\title{
EL TRATADO DE NIZA. ENTRE LA CONSOLIDACIÓN DE LA UNIÓN EUROPEA DE MAASTRICHT Y EL DEBATE SOBRE EL FUTURO DE EUROPA
}

\section{Elena F. PÉREZ CARRILLO*}

RESUMEN: Las principales reformas introducidas en el Tratado de Niza incluyen las cooperaciones reforzadas, cuestiones institucionales y la ampliación del uso de mayorías calificadas. El sistema de cooperaciones reforzadas, introducido en el Tratado de Amsterdam, fue fortalecido en Niza y permitirá una integración más rápida y profunda. La reforma institucional tocó el número de votos asignados en el Consejo Europeo, y la distribución de asientos en el Parlamento Europeo y en la comisión. En cuanto a la Corte de Justicia, varias de sus características podrán ser reformadas con el voto unánime del consejo en lugar de requerirse una Conferencia Intergubernamental. Ha habido cambios en la distribución de las competencias entre el Tribunal de Primera Instancia y la Corte de Justicia. Esta última será integrada por un juez por cada Estado miembro, y trabajará preferentemente en salas, y no en pleno como en el pasado.

ABSTRACT: The main reforms introduced in the Treaty of Nice include reinforced cooperation, institutional matters, and the widening of the resort to qualified majority. The system of reinforced cooperation, introduced in the Treaty of Amsterdam, was strengthened in Nice and will enable a faster and deeper process European integration. Institutional reform addressed the number of votes assigned in the European Council, and the allocation of seats in the European Parliament and the Commission to each country. As regards the Court of Justice, many of its features will be now subject to reform only with the unanimous vote in the Council, rather than through an Intergovernmental Conference. There have been changes in the distribution of competence between the Court of First Instance and the Court of Justice. The latter is now integrated by one judge per country, and it will work preferably in Chambers rather than in plenary sessions.

RESUMÉ: Les réformes principales introduites dans le Traité de Nice incluent les coopérations renforcées, les questions institutionnelles et la plus grande utilisation de la majorité qualifiée. Le système de coopération renforcée, introduit dans le Traité d'Amsterdam, a été fortifié à Nice et permettra une intégration plus rapide et profonde. La réforme institutionnelle aborde le nombre de votes attribué dans le Conseil Européen et la distribution de sièges dans le Parlement Européen et dans la Commission. Par rapport à la Cour de Justice, de nombreuses caractéristiques pourront être réformées avec la mise en place du vote à l'unanimité des membres du Conseil au lieu de convoquer une conférence intergouvernementale. Il y a eu des modifications dans la répartition des compétences entre le Tribunal de première instance et la Cour de Justice. Cette dernière se verra attribuer un juge pour chaque État membre, et elle travaillera de préférence en chambres et non en session plénière comme par le passé.

\footnotetext{
* Universidad de Santiago de Compostela.
} 
SUMARIO: I. Antecedentes. II. La firma del tratado. III. La estructura formal del tratado. IV. Las principales reformas introducidas en Niza. V. Perspectivas.

\section{ANTECEDENTES}

Una vez más, como ya ocurriera en la firma del Tratado de Amsterdam en junio de 1997, las primeras reacciones a la cumbre de diciembre de 2000 en la que se concluyeron las negociaciones del Tratado de Niza han sido las voces críticas, que no se han hecho esperar. Entre los comentaristas insatisfechos con los resultados obtenidos destaca el presidente de la comisión Romano Prodi. ${ }^{1}$ Otros han querido ver que formalmente la Conferencia Intergubernamental de 2000 ha cumplido los objetivos de reforma institucional que permita hacer frente a las inminentes adhesiones ${ }^{2}$ para la que fue convocada en primer término, o se inclinan a una interpretación "evolutiva" de los recientes tratados, que se enmarcarían en un momento histórico reconstitutivo especial ${ }^{3}$ y específico de la Unión Europea iniciado en Maastricht que debe ahora evolucionar, ${ }^{4}$ entre estos últimos no son desdeñables las posturas del Parlamento Europeo, que por

1 El presidente Romano Prodi se manifestó tras la conclusión de la citada cumbre. Pueden consultarse sus declaraciones en las hemerotecas, secciones generales y especializadas. Entre otras citamos la entrevista "El Tratado de Niza resultó decepcionante", Boletín Europeo de la Universidad de la Rioja, núms. 7 y 8, marzo de 2001, pp. 3 y ss. También, en el mismo boletín, véase el artículo editorial: Martín y Pérez de Nanclares, J., "La reforma de Niza: compleja, insuficiente, provisional e incierta".

2 En este sentido, Best, Edward, “The Treaty of Nice: Not Beautiful but It'll Do", Eipascope, 2000/1, Institut Européen d'Administration Publique. Sobre los objetivos de reforma cara a la ampliación: Louis, Jean Victor, "La réforme des institutions de l'Union européenne", Revue du Marché Commun, núm. 4433 de diciembre de 2000.

3 Esta apreciación respondería a un proceso, que ya desde 1993 consideraron algunos como J. Weiler, que correspondía a un "momento constitucional". Respecto del reflejo de esta situación, es particularmente interesante la visión de Aldecoa Luzurraga, F., "La apertura del proceso constituyente”, Boletín Europeo de la Universidad de la Rioja, núms. 7 y 8 , pp. 7 y ss.

4 El Parlamento Europeo reunido en sesión plenaria el día 31 de mayo de 2001, no se manifestó expresamente a favor de recomendar a los parlamentos nacionales de los Estados miembros el voto a favor del tratado. En cambio, reclamaron la Convocatoria de una Convención para preparar la próxima reforma de Niza. Según el europarlamentario español popular Íñigo Méndez de Vigo, el texto representa una "época que comienza en Maastricht y que termina aquí", propugnando un cambio en la negociación de los tratados, en el sentido de abanderar el nivel estrictamente intergubernamental, que ha presidido las reformas hasta el momento, para sustituirlo por un régimen de Convención que comience sus trabajos a principios del año 2002, y que se componga por parlamentarios nacionales, europarlamentarios y miembros de la comisión y representantes de los Estados miembros. El europarlamentario socialista portugués recomendó que tal convención sea convocada en el próximo Consejo Europeo de Gotteburgo. 
primera vez no ha recomendado expresamente a los parlamentos nacionales la ratificación del acuerdo negociado en Niza, sino que se ha fijado sobre todo en las perspectivas de la nueva reforma prevista para el año 2004, o las declaraciones públicas de los dirigentes europeos en el marco del debate abierto sobre el futuro de Europa. ${ }^{5}$ Por el momento, la reacción más contundente, y la que promete ser objeto de más profundas negociaciones, ha sido el "no" de Irlanda en el referéndum de 8 de junio de 2001. Este resultado plantea la necesidad a corto plazo de encontrar nuevas vías para despejar la eventual entrada en vigor del tratado y la ampliación. ${ }^{6}$

El Tratado de Amsterdam, fruto en gran medida de los "flecos" que quedaron abiertos en Maastricht, ${ }^{7}$ ya reconocía en su propio texto la necesidad de continuar las reformas iniciadas en él. ${ }^{8}$ Uno de sus protocolos señalaba que, con vistas a la próxima ampliación de la Unión Europea se deberían realizar algunas reformas institucionales ideadas en aquel momento en dos etapas que correspondían al sistema de ampliación previsto entonces:

- Modificaciones que debían estar aprobadas en el momento de entrada en vigor de la primera ampliación de la Unión Europea. A partir de ese momento se preveía que la comisión comprendería un nacio-

5 Las declaraciones abundan en los periódicos en estos días. Desde las propuestas alemanas tendentes a una "federalización" que clarifique el reparto de competencias, aún a costa de reducir o delimitar más estrictamente que en la actualidad las comunitarias; a las francesas, apoyadas entre otros por Portugal y algunos países "pequeños", de reforzar el papel de la comisión y que propone una Europa de Estados-Nación.

6 En el momento de escribir estas líneas, sólo el Parlamento danés ha ratificado el Tratado de Niza. Por ahora, se han planteado tres posibles vías de solución al "no irlandés": El intento de una nueva CIG, solución que no parece que vaya a ser adoptada, la modificación de la Constitución irlandesa para suprimir la necesidad de consultas populares, y por último seguir el modelo planteado al producirse en 1992 la negativa danesa a dar su apoyo a la ratificación del Tratado de Maastrich. De adoptarse la solución de nueva negociación con Irlanda y nuevo referéndum, parece imposible replantear lo que seguramente constituye una piedra angular en la negativa irlandesa: el nuevo reparto de poder. En cambio, la PESC y la participación de las tropas de este país podría utilizarse como tabla de "salvamento" o soporte de una nueva negociación y de un nuevo referéndum.

7 Como se recordará, el Tratado de Amsterdam era una consecuencia directa de las exigencias del artículo N2 del Tratado de Maastricht que exigía la convocatoria en 1996 de una Conferencia Intergubernamental para la reforma.

8 La dificultad en la ratificación del Tratado de Maastrich no daba pie a cerrar todas las cuestiones abiertas. En septiembre de 1992, Miterrand lo sometió a consulta popular en Francia, donde vencieron los partidarios por un estrecho margen de $51 \%$ frente a los oponentes con un $48,4 \%$. En Dinamarca, un primer referéndum en 1992 dio la victoria a los que se oponían a la ratificación, si bien después de negociaciones con Dinamarca y un segundo referéndum en mayo de 1993, cambió la situación. 
nal de cada uno de los Estados miembros, siempre que ya se hubiese modificado la ponderación de votos en el seno del consejo.

- Modificaciones previstas para cuando el número de miembros de la Unión Europea estuviese a punto de superar el de 20. Al menos con un año de anterioridad se debería reunir una nueva Conferencia Intergubernamental para llevar a cabo una revisión global de los preceptos de los tratados en la composición y en el funcionamiento de las instituciones.

La reunión del Consejo Europeo de Colonia de 3 y 4 de junio de 1999 decidió la convocatoria de una Conferencia Intergubernamental ${ }^{9}$ prevista para reunirse a comienzos de 2000 para resolver los asuntos que habían quedado pendientes en Amsterdam, previa consulta al Parlamento Europeo, de acuerdo con el artículo 48 del Tratado de la Unión Europea. ${ }^{10}$ El Consejo Europeo de Helsinki de diciembre de 1999 confirmó el mandato a la conferencia, pero además dio un nuevo impulso al proceso de ampliación que modificaba el sistema previsto hasta entonces en virtud de que se habían hecho dos bloques de países candidatos para efectuar negociaciones tendentes a la ampliación escalonada. A partir de las conclusiones de Helsinki, se reúnen en un marco único los 13 países candidatos, sin perjuicio de que las negociaciones se realizasen por separado, y sin perjuicio de que eventualmente el proceso de entrada en la Unión Europea dependiera de la velocidad con la que cada uno de los candidatos estuviese en situación de adherirse. Esto significaba que, al menos teóricamente, podría tener una ampliación global que desde el principio superase los 20 miembros. Por lo tanto, las etapas previstas en Amsterdam para la adaptación de las instituciones perdían gran parte de su sentido.

El Consejo Europeo, durante la Presidencia finlandesa, señaló tres aspectos concretos sobre los que ésta debería concentrar sus esfuerzos: el tamaño y la composición de la comisión, el peso y balance de votos en el seno

9 Puede decirse que el método seguido por la CIG para la elaboración del Tratado de Niza se encuentra a medio camino entre el sistema de Maastricht y el de Niza. El primero se negocio a partir de un texto básico que fue modificándose. Es el sistema más tradicional de negociación de tratados internacionales. El segundo se negoció sobre la base de textos variados acompañados de numerosas propuestas de cada delegación.

10 A este respecto, véase la resolución del Parlamento Europeo sobre la convocatoria de la Conferencia Intergubernamental (14094/1999-C5-0341/1999-1999/825 (CNS), y el informe de la Comisión de Asuntos Institucionales A5-0018/2000. 
del consejo y la posible ampliación de la aplicación del voto por mayoría cualificada en el consejo, y otras modificaciones.

Una serie de trabajos fueron muy importantes en la definición de los debates que se suscitaron a lo largo de la práctica totalidad del año 2000, y que crearon un estado de opinión tanto en los ámbitos políticos jurídicos responsables de la reforma, como entre el público en general:

- El documento Dehaene presentado el 10 de octubre de 1999 por el ex primer ministro de Bélgica. ${ }^{11}$

- El discurso de Joschka Fisher ${ }^{12}$ que reconoció y expresó públicamente la escala de asuntos que "están en juego" y que abrió un debate público no sólo entre los líderes europeos, ${ }^{13}$ sino además entre los mismos ciudadanos. ${ }^{14}$

- Las diversas contribuciones de la comisión en preparación de la Presidencia de la UE. ${ }^{15}$

- Los trabajos sobre la reforma del Tribunal de Justicia. ${ }^{16}$

11 Weizsäcjker, Richard von; Dehaene, Jean-Luc y Simon, David, Las implicaciones institucionales de la ampliación. Informe de la Comisión Europea.

12 Thoughts on the finality of European integration. Discurso en la Universidad Humboldt de Berlín el 12 de mayo de 2000.

13 Entre los que primero respondieron a Fischer, estuvo el presidente francés Chirac en un discurso en el Bundestag, el Parlamento alemán. Josín, primer ministro francés, también reaccionó no sólo frente a la propuesta del ministro alemán, sino además ante la inesperada alocución de su presidente gaullista.

14 Las hemerotecas muestran cómo durante el verano de 2000, todos los medios de comunicación europeos se hacían eco de las distintas declaraciones sobre el modelo más oportuno para la Unión Europea, debate al que se fueron uniendo los editores mediáticos, comentaristas y los propios ciudadanos.

15 "Adaptar las instituciones para que la ampliación sea un éxito", Dictamen de la comisión en virtud del artículo 48 del Tratado de la Unión Europea sobre la reunión de una conferencia de los representantes de los gobiernos de los Estados miembros con el fin de modificar los tratados, 26 de enero de 2000 COM (2000) 34. Otras contribuciones de la comisión también resultaron de gran interés como las comunicaciones: "Adaptar las instituciones para realizar la ampliación con éxito" de 2 de diciembre de 1999 COM (1999) 592 final; "Un tratado fundamental para Europa" COM (2000) 434 final; "Contribución suplementaria de la comisión a la Conferencia Intergubernamental sobre las reformas institucionales: el estatuto de los partidos políticos COM (2000) 444 final; la comunicación de la comisión a la Conferencia Intergubernamental sobre la reforma de las instituciones COM (2000) 771 final; o la comunicación de la comisión. Contribución suplementaria de la comisión a la Conferencia Intergubernamental sobre las Reformas Institucionales, votación por mayoría cualificada en los aspectos relacionados con el mercado único de los ámbitos de la fiscalidad y la seguridad social, documento COM (2000) 114 final. También ciertos documentos internos como Commission Reform Strategic Options Paper. Communication from Kinnock, in agreement with Mrs Schreyer and President Prodi, to the Commission, 1999, entre otros.

16 Destacan el Documento de Reflexión sobre el Futuro del Sistema Jurisdiccional de la Unión Europea, presentado por el presidente del Tribunal de Justicia ante los ministros de Justicia reunidos 
La agenda de temas señalada en la Presidencia a fines del segundo semestre de 1999 quedó abierta a posibles adiciones. El 14 de febrero de 2000 se abrió solemnemente la CIG. Los ministros y representantes gubernamentales del más alto nivel se reunieron cada 15 días aproximadamente, según el calendario provisional accesible en Internet a lo largo del periodo de la conferencia en una página Web habilitada dentro del servidor interinstitucional europa.eu.int, y en el marco del diálogo con los ciudadanos, relacionado con el movimiento a favor de una mayor transparencia en el seno de la Unión Europea. El periodo de negociaciones en el seno de la conferencia puede dividirse en dos: ${ }^{17}$

- La primera parte correspondió con la Presidencia portuguesa de la Unión Europea, una etapa de exploración de los diversos puntos de vista. El tema más debatido fue la extensión el uso de mayorías cualificadas ${ }^{18}$ y con ello la ampliación de la codecisión. A efectos de facilitar el acuerdo, la Presidencia confeccionó un listado de materias que hasta entonces se decidían por unanimidad y que podrían pasar a resolverse por mayorías cualificadas. En el Consejo Europeo de Feira en junio del año 2000, se ampliaron los temas objeto de trabajo en la Conferencia Intergubernamental de 2000, por lo que se refiere fundamentalmente a las cooperaciones reforzadas y a la PESC. Otros asuntos se discutieron separadamente, entre ellos la composición del Tribunal de Justicia Europeo, debatido en trono a estudios específicos del propio tribunal, del grupo amigos de la Presidencia y la comisión, y cuyas bases ya quedaron fijadas en mayo de 2000.

- En la segunda etapa, correspondiendo con la Presidencia francesa, se identificaron tres tipos de procesos: Por una parte los que podían

en el Consejo de Ministerios en Bruselas el 27 y 28 de mayo de 1999; el Informe del Grupo de Reflexión sobre el Futuro del Sistema Judicial de las Comunidades Europeas de enero de 2000, elaborado bajo el antiguo presidente del tribunal señor Due, o la Contribución del Tribunal de Justicia y del Tribunal de Primera Instancia a la Conferencia Intergubernamental de 25 de febrero de 2000.

17 Yataganes, X. A., "The Treaty of Nice: The sharing of power and the institutional Balance in the European Union. A Continental Perspectiva”, Jean Monnet Working Papers, núm. 1, 2001 pp. 1-3.

18 Entre las cuestiones que finalmente quedaron excluidas del voto por mayoría cualificada se encuentran la fiscalidad y la seguridad social. En este sentido, es importante el documento de la comisión: COM (2000) 114 final de Comunicación de la Comisión, Contribución Suplementaria de la Comisión a la Conferencia Intergubernamental sobre las Reformas Institucionales, votación por mayoría cualificada en los aspectos relacionados con el mercado único de los ámbitos de la fiscalidad y la seguridad social. 
mantenerse al margen de las discusiones finales y para los que era posible alcanzar acuerdos antes de la cumbre de Niza. Otros eran los temas centrales, respecto de los que los representantes permanentes tenían el mandato de "llegar tan lejos como fuera posible", entre los que se encontraba el equilibrio de poderes (composición de la comisión, valor relativo de los votos del consejo etcétera), que podrían resolverse por parte de los jefes de Estado y de gobierno. Por último existían otras cuestiones de interés como la reorganización de los tratados, los partidos políticos europeos, el establecimiento de un fiscal europeo, la clarificación de las bases jurídicas para la constitución de agencias europeas, la jerarquía normativa de la UE, y sobre todo la Carta de Derechos Fundamentales y la accesión de UE al convenio europeo para la protección de los derechos humanos y libertades fundamentales.

El 3 de noviembre, la Presidencia presentó un borrador de tratado en el que dejaba abiertos tres capítulos: la composición de la comisión, el reparto de los miembros del Parlamento entre los Estados de la Unión Europea (sobre el número máximo de 700 fijado en el Tratado de Amsterdam, superado en Niza con 732) y la ponderación de votos en el seno del consejo.

\section{LA FIRMA DEL TRATADO}

El tratado fue acordado en el Consejo de Niza, el 7 y el 8 de diciembre de 2000. A continuación, una versión provisional quedo disponible en Internet, pendiente, según rezaba el texto, de una corrección lingüística. El 26 de febrero de 2001 el Tratado de Niza fue firmado por los representantes plenipotenciarios de los Estados miembros de la Unión Europea, abriéndose el proceso de ratificación según los diversos sistemas constitucionales de cada país. El texto aprobado fue publicado en el Diario Oficial de las Comunidades Europeas, serie C, número 80, de 10 de marzo de 2001.

En el momento de escribir estas líneas, el pueblo irlandés acaba de pronunciarse en contra de la ratificación en un referéndum que tuvo lugar el día 8 de junio de 2001. Únicamente el Parlamento danés ha ratificado, mientras que otros como el español o el francés se encuentran en trámite de hacerlo. 
Como primera apreciación, cabe recordar que la declaración 23 del tratado, ya prevé una nueva reforma a través de una conferencia que se convocará en 2004, precedida por un amplio debate sobre el futuro de Europa que exceda los límites de la CIG, que abarca a los ciudadanos ${ }^{19}$ así como a los principales líderes europeos. ${ }^{20}$ Frente a 30 primeros años de desarrollo de la Unión Europea, en que no tuvieron lugar transformaciones sustantivas del derecho originario (con alguna excepción como los tratados de 1965 y 1970 sobre recursos propios y poderes del Parlamento Europeo), a partir de 1985 estamos ante un proceso de cambio casi continuo. Una vez que se supere la fase de ratificaciones del Tratado de Niza, quedarán aún abiertas muchas cuestiones,$^{21}$ algunas de las cuales se fijan ya en la citada declaración 23 , a la que nos referimos más adelante.

\section{LA ESTRUCTURA FORMAL DEL TRATADO}

Formalmente el Tratado de Niza se divide en varias secciones. En la primera se relacionan los jefes de Estado que convienen el tratado y los plenipotenciarios, generalmente ministros de asuntos exteriores, que rubrican el texto. El resto del documento consta de dos partes, cuatro protocolos, un acta final, las 24 declaraciones adoptadas, y las tres declaraciones de las que tomó nota la conferencia.

La primera parte, titulada "modificaciones sustantivas" consta de seis artículos, cada uno de ellos dedicado a la modificación de alguno de los tratados de derechos originario europeo: el Tratado de la Unión Europea (artículo 1o.), El Tratado de la Comunidad Europea (artículo 2o.), el

19 En este sentido, ya se ha puesto en marcha el "Diálogo con los ciudadanos" accesible desde el servicio institucional de la Unión Europea.

20 Las declaraciones de los líderes europeos refuerzan la idea de que estamos ante un momento de cambio "constitucional". Romano Prodi, quien se ha manifestado por la necesidad de que Europa cuente con un gobierno económico, idea también favorecida por Jospín en relación con la zona euro que se ha manifestado a favor de una federación de "Estados Nación"; Schoröder habla de devolver competencias a los Estados en ámbitos como la agricultura y la cohesión, Blair se opone a la idea de una Constitución escrita, etcétera. Sobre estas cuestiones recomendamos una revisión de las hemerotecas en las fechas posteriores a la firma del Tratado de Niza, desde marzo hasta junio de 2001, en particular.

21 El hecho de que hayan quedado nuevos "flecos" ha sido criticado. Las críticas han sido tan abundantes que sólo referimos alguna por el interés que nos ha suscitado. Un reciente artículo de Alonso García, R., "Nuestro futuro, ¿cuestión de juristas?”, El País, 24 de abril de 2001, p. 12, sección de Opinión; en relación con todas estas cuestiones ("los nuevos flecos de Niza") y en particular con la necesidad de la simplificación de los tratados, que para algunos es sólo un asunto técnico, y que sin embargo el autor considera muy relevante. 
Tratado Euratom (artículo 3o.), el Tratado CECA (artículo 4o.), el Protocolo sobre los Estatutos del Sistema Europeo de Bancos Centrales y del Banco Central Europeo (artículo 5o.), y el Protocolo sobre las Inmunidades y Privilegios de las Comunidades Europeas (artículo 6o.).

La segunda parte se dedica a Disposiciones transitorias y finales. A continuación se insertan los cuatro protocolos del Tratado de Niza que constituyen anexos con el mismo rango jerárquico que el cuerpo principal:

- El referente a la ampliación de la Unión Europea, que supone la derogación del Protocolo sobre las instituciones de Amsterdam, y el nuevo reparto de representantes en el Parlamento Europeo, el cambio en la ponderación de votos del consejo, y nuevas disposiciones sobre la comisión, asuntos a los que nos referimos más adelante.

- El protocolo sobre el Estatuto del Tribunal de Justicia, con el Estatuto de jueces y abogados generales, organización y procedimiento, que también será objeto de reflexión en nuestro siguiente apartado.

- El Protocolo sobre las consecuencias financieras de la expiración del Tratado CECA y el Fondo de investigación del Carbón y el Acero, en virtud del cual a partir del día 24 de julio de 2002 todos los elementos del patrimonio activo y pasivo de la CECA serán transferidos a la Comunidad Económica Europea y se destinarán a la investigación en los sectores del carbón y del acero, primero con la denominación "CECA en liquidación” y posteriormente con el nombre "Activos del Fondo de Investigación del Carbón y del Acero". Estos fondos de investigación se administrarán de forma independiente y la margen del Programa Marco plurianual de $\mathrm{I}+\mathrm{D}$, que de conformidad con el artículo 166 TCE rija en cada momento en la CE. La adopción de decisiones respecto de este fondo no se define directamente según los sistemas clásicos de "directivas-reglamento, etcétera", sino que se prevé que la decisión unánime del consejo, previa consulta al Parlamento y a propuesta de la comisión, servirá para adoptar acuerdos incluido el que fije cuáles sean los instrumentos más adecuados para la adopción de decisiones sobre tal fondo. Se hace referencia a unos tipos de instrumentos decisorios específicos para este fondo:

a) Principios esenciales.

b) Directrices financieras plurianuales de gestión del fondo.

c) Directrices técnicas de investigación. 
- El protocolo sobre el artículo 67 del Tratado CE, es decir, sobre la coordinación entre los Estados, y entre éstos y la comisión en lo referente a la libre circulación de personas, visados, asilo e inmigración a partir del día 1 de mayo de 2004, generaliza la mayoría cualificada en el seno del consejo. Excluye, frente a lo que se había acordado en Amsterdam, la necesidad de una nueva decisión unánime del consejo sobre el método de adopción de decisiones en los asuntos referentes a la coordinación y cooperación entre Estados y comisión en lo referente al título IV CE.

El acta final del tratado recoge, como es habitual, un elenco nominativo de la totalidad de los textos adoptados: El propio tratado de Niza por el que se modifican el Tratado de la Unión Europea, los tratados constitutivos de las comunidades europeas y actos conexos, los protocolos, y las declaraciones.

Por lo que respecta a las declaraciones adoptadas por la conferencia, son 24, y entre ellas destacamos algunas:

- La primera se refiere a la PESC en incide en la voluntad política de acelerar la puesta en marcha de los mecanismos acordados en el Consejo Europeo de Niza de diciembre de 2000, sin necesidad de esperar a su entrada en vigor. En este sentido resultan destacables una serie de textos aprobados el 22 de enero de 2001 y publicados en el Diario Oficial L 27 de 2001:

a) La decisión por la que se crea el Comité Político y de Seguridad (2001/78/PESC) $)^{22}$ respecto a cuyo papel remite al anexo III del informe de la Presidencia del Consejo Europeo de Niza.

b) La Decisión por la que se crea el Comité Militar de la Unión Europea (2001/79/PESC). ${ }^{23}$

22 La creación de este comité ya estaba prevista en el TCE. El artículo 25 del TUE, sin perjuicio del artículo 207, dice: "un comité político seguirá la situación internacional en los ámbitos de política exterior y de seguridad común y contribuirá a definir la política mediante la emisión de dictámenes". El Consejo Europeo de Helsinki dio el fundamento político para la constitución de un comité político y de seguridad provisional en el año 2000, y el Consejo de Niza acordó la creación de tal comité con carácter permanente, para estar enterado de la situación internacional en los aspectos relativos a la PESC, para contribuir a la formulación de políticas mediante dictámenes, ofrecer orientaciones a los demás comités, ser interlocutor preferente del secretario general, alto representante y representantes especiales, dar directrices al comité militar, etcétera.

23 El comité militar se integra por los jefes de Estado mayor de la Defensa, representado por sus delegados militares o se reunirá a nivel de jefes de Estado mayor, cuando sea necesario. 
c) La Decisión relativa a la creación del Estado Mayor de la Unión Europea (2001/80/PESC) que formará parte de la Secretaría General del consejo.

- La declaración sobre el artículo 31.2 del TUE, es decir sobre el título VI del TUE (cooperación policial y judicial en materia penal), recuerda que la Red Judicial Europea ya ha sido creada en virtud de la Acción Común 98/428/JAI (DOL 191), y que la decisión política sobre la creación de un Eurojust o unidad compuesta por jueces, fiscales, jueces o funcionarios para la coordinación en la lucha contra el delito, está prevista desde el Consejo Europeo de Tampere de octubre de 1999.

- La declaración sobre el artículo 10 matiza y completa el sentido de tal artículo, en particular por lo que respecta a la necesaria "cooperación leal" entre las instituciones comunitarias, y a la circunstancia de que las instituciones, que pueden celebrar acuerdos entre ellas requieren del consentimiento de Parlamento, consejo y comisión para realizarlos, y deben entender que tales acuerdos no podrán en ningún caso alterar el alcance, ni completar el tratado.

- La declaración sobre el artículo 21.3 insta a las instituciones a responder a las peticiones de los ciudadanos en el periodo más breve posible a las consultas o peticiones que éstos les puedan formular.

- La declaración relativa al artículo 67 supone la introducción de la codecisión a partir del 1 de mayo de 2004 para las medidas (dentro del título IV) que establezcan las condiciones de viaje en el territorio de la Unión Europea por parte de nacionales de terceros países, durante un periodo no superior a tres meses, así como en materia de inmigración por lo que respecta a la inmigración y residencia ilegales, incluida la repatriación de residentes ilegales, así como sobre los controles a realizar en las fronteras exteriores (una vez que exista un acuerdo sobre el ámbito de aplicación de medidas relativas al cruce de personas por las fronteras exteriores).

- La declaración relativa al artículo 111 TCE se refiere a que todos los Estados de la zona europea deben poder participar plenamente en la preparación de posiciones de la comunidad a escala internacional, cuando éstas tengan interés para la unión económica y monetaria.

- La declaración relativa al artículo 175 TCE manifiesta la voluntad de los Estados de que la Unión Europea tenga una función impulsora en la protección del medio ambiente en todo el mundo y que de- 
berán desarrollarse todas las posibilidades que ofrece el tratado con tal fin, tanto por lo que se refiere a incentivos e instrumentos de mercado, como al fomento del desarrollo sostenible.

- La declaración relativa al artículo 191 TCE clarifica que ese artículo (sobre los partidos políticos europeos y su financiación) no afecta a las normas constitucionales nacionales.

- Las declaraciones sobre el artículo 225 TCE (números 12 a 16) invitan al Tribunal de Justicia y a la comisión a efectuar un estudio global sobre el reparto de competencias entre el TJCE y el Tribunal de Primera Instancia y a presentar propuestas al respecto. Señalan ciertas disposiciones que, a juicio de la conferencia, deberían formar parte del Estatuto del tribunal: el papel de las partes en el procedimiento ante el TJCE, para garantizar la salvaguardia de sus derechos; el efecto del procedimiento de reexamen sobre el carácter ejecutivo de la resolución del TPI; y el efecto de la resolución del TJCE. Fijan un plazo deseable de un máximo de tres años desde la entrada en vigor del Tratado de Niza para la evaluación de la reforma del sistema jurisdiccional (declaración 14), establecen la conveniencia de recurrir al procedimiento de urgencia cuando el TJCE reexamine cuestiones prejudiciales decididas por el TPI (declaración 16).

- La declaración número 20 incluye una serie de cuadros sobre el reparto de escaños en el Parlamento Europeo, sobre la ponderación de votos en el consejo, sobre el Comité Económico y Social y el Comité de las Regiones que serán aplicables en las conferencias de adhesión. Son tablas realizadas sobre la base de una unión de 27 Estados, y en la que se ha tenido en cuenta sólo los países candidatos que han iniciado efectivamente las negociaciones tendentes a la adhesión, y a los actuales miembros. Esta declaración contiene además una referencia a las triples mayorías necesarias para la adopción de decisiones en un consejo de 27 miembros. Esta declaración se completa con la número 21 relativa al umbral de la mayoría cualificada y al número de votos de minoría de bloqueo en una unión ampliada. Las nuevas ponderaciones entrarán en vigor el 1 de enero de 2005 y el umbral de la mayoría cualificada irá evolucionando a medida de que se añadan nuevos miembros. Al analizar las principales reformas introducidas en el derecho originario europeo en Niza volveremos a incidir sobre estas cuestiones. 
- La declaración 22 se refiere al lugar de reunión de los Consejos europeos. Establece que a partir de 2002, una reunión del Consejo Europeo por Presidencia se celebrará en Bruselas, y que, a partir del momento en el que la Unión Europea conste de 18 miembros, todas las reuniones tendrán lugar en dicha ciudad.

- La declaración 23 o relativa al futuro de la Unión Europea constituye una primera proyección hacia una unión ampliada. Prevé la apertura inmediata de un gran debate sobre el futuro, en el que además de la participación de los Estados y de las instituciones europeas debe participar toda la opinión pública europea, desde los parlamentos nacionales, las universidades, los representantes de la sociedad civil y los propios ciudadanos. Establece un calendario aproximado que incluye que en diciembre de 2001 la reunión del Consejo de Laaken realizará una declaración con iniciativas para la continuación del proceso. Dentro del programa de debate provisional fija algunos apartados esenciales: la delimitación de competencias entre los Estados y la Unión Europea, teniendo en cuenta el necesario respeto del principio de subsidiariedad; el Estatuto de la Carta de Derechos Fundamentales proclamada en Niza; la simplificación y clarificación de los tratados y la función de los parlamentos nacionales en la Unión Europea, todo ello dentro de la necesidad de profundizar en la trasparencia y en la legitimidad democrática de la Unión Europea. Por último, señala que en 2004 se reunirá una nueva Conferencia Intergubernamental para valorar las conclusiones del debate, sin que tal conferencia suponga un prerrequisito para la ampliación.

- Por último, la declaración 24 sobre la CECA insiste en la necesidad de mantenimiento del sistema estadístico de la CECA, una vez expirado el Tratado sobre la Comunidad del Carbón y del Acero.

Por lo que respecta a las declaraciones de las que tomó nota la conferencia, se reducen a tres. En la primera, el gobierno de Luxemburgo se compromete a no reivindicar la sede de las salas de recurso de la Oficina de Armonización del Mercado Interior de Alicante, incluso si éstas se convierten en salas jurisdiccionales. ${ }^{24}$ En la segunda, Grecia, España y Portugal afirman que su acuerdo para que las decisiones del artículo 161

24 Esta declaración se efectúa sin perjuicio de la Declaración de 8 de abril de 1965 sobre la sede de las instituciones, organismos y servicios futuros que hubiera permitido a Luxemburgo reclamar todas las salas jurisdiccionales. 
TCE (funciones, objetivos y organización de los fondos con finalidad estructural y del fondo de cohesión) se realicen por mayoría cualificada, se basa en el entendimiento de que las perspectivas financieras aplicables a partir del 1 de enero de 2007 tengan idéntica duración a las actuales. Frente a dicha declaración, Dinamarca, Alemania, Países Bajos y Austria declaran que la posición de Grecia, España y Portugal no tiene como efecto prejuzgar la acción de la comisión ni su derecho de iniciativa.

En la última sección del tratado se inserta la fecha (Niza a 23 de febrero de 2001) y las rúbricas efectuadas por los plenipotenciarios en nombre de sus respectivos jefes de Estado, que en el caso de Bélgica va acompañada de la declaración expresa de que la misma vincula a las varias comunidades de este país: la comunidad francesa, la flamenca, la germanófona, la región balona, la región flamenca y la región de BruselasCapital.

\section{LAS PRINCIPALES REFORMAS INTRODUCIDAS EN NIZA}

Las principales y más profundas reformas introducidas por este tratado han sido las cooperaciones reforzadas y las cuestiones institucionales, seguidas de una ampliación en el uso de la mayoría cualificada y la codecisión.

A pesar de las críticas suscitadas, al menos formalmente, el texto aprobado ha cumplido el objetivo de reforma institucional suficiente para permitir la ampliación, siempre, claro está, que los ciudadanos europeos decidan apoyar a través de los respectivos sistemas nacionales de ratificación, la eventual entrada en vigor de este tratado.

\section{Las cooperaciones reforzadas}

Como veíamos, las cooperaciones reforzadas se introdujeron en la Agenda de la CIG en el Consejo Europeo de Feira de junio de 2000. Originalmente fueron una aportación del Tratado de Amsterdam como consecuencia de la necesidad de combinar el deseo o la necesidad de algunos países de avanzar con mayor rapidez o profundidad que otros en el proceso de integración europea, lo que se había hecho particularmente en 
la etapa de negociación y de ratificación del Tratado de Maastricht o primer Tratado de la Unión Europea. ${ }^{25}$ En Niza se profundiza y ordena este proceso.

En Amsterdam era necesaria una mayoría de Estados para configurar una cooperación de este tipo. El número mínimo para establecer una cooperación se fija ahora en ocho, independientemente del de Estados miembros de la UE, con lo que una vez ampliada la UE, decaerá la necesidad de que se trate de una mayoría de Estados.

El procedimiento de adopción de decisiones se clarifica. Por lo que respecta a la creación de cooperaciones reforzadas, la petición de los Estados miembros se dirige a la comisión para que ésta presente una iniciativa, o bien no lo haga justificando su posición. Este sistema será aplicable no sólo a las cooperaciones del pilar comunitario, sino también respecto de las del tercero. Es decir, que la comisión adquiere un poder de iniciativa que no tenía en este ámbito; excepto cuando la comisión decida no presentar la propuesta, supuesto en el que los Estados miembros pueden dirigirse directamente al consejo solicitado autorización, de conformidad con el nuevo artículo 40 a(1) del TUE.

La decisión sobre el establecimiento de una cooperación reforzada corresponde, por mayoría cualificada al consejo, y en el caso de que se trate de una materia que se rija por el procedimiento de cooperación requerirá el dictamen conforme del Parlamento Europeo. A petición de cualquier miembro del consejo, el asunto se eleva al Consejo Europeo, que decidirá, devolviendo el expediente al consejo para que adopte formalmente el acto correspondiente. Se reitera la necesidad de que los actos y contenidos en el marco de las cooperaciones reforzadas respeten el tratado. El procedimiento se ha simplificado, respecto del texto de Amsterdam, pues parece reconducir el método de adopción de decisiones al general: iniciativa comisión-consejo-parlamento. En Amsterdam se requería de una autorización previa del consejo, además la elevación al Consejo Europeo exigía de una mayoría cualificada. El sistema de Niza es más simple, elimina el veto en el consejo, y no se menciona la unanimidad en el seno del Consejo Europeo. A cambio, la decisión en el conse-

25 Sobre las cooperaciones reforzadas nos manifestamos sucintamente en Pérez Carrillo, E. F., "Más allá del mercado único. Algunas aportaciones del Tratado de Amsterdam al lento proceso de integración europea", Revista de Estudios Europeos, núm. 22, 1999, pp. 69-88, y en particular pp. 70 y 71; y en "El Tratado de Amsterdam. Entre el mercado de los quince y el siglo XXI", Noticias de la Unión Europea, núm. 189, 2000, pp. 171-180, en particular pp. 173 y 174. 
jo requiere el consenso, ya que permite que cualquier Estado solicite la elevación al Consejo Europeo, sin necesidad de alegar ningún tipo de razón en concreto.

Por lo que respecta al proceso de adhesión a una cooperación reforzada, no parece que el sistema se haya modificado en exceso. Uno de los cambios consiste en que la decisión de la comisión puede establecer modalidades particulares respecto de la adhesión a esa cooperación, que sigue planteándose como un sistema abierto a la participación de todos los Estados miembros de la Unión Europea. Los plazos se mantienen: la voluntad de adherirse es comunicada a la comisión. En el plazo de tres meses, desde la recepción, la comisión emite un dictamen al consejo y en el plazo de cuatro meses (decide en la versión de Amsterdam, o) se pronuncia según el texto de Niza.

A partir de la entrada en vigor del Tratado de Niza, las cooperaciones reforzadas serán posibles en el ámbito de la PESC, es decir en el segundo pilar anteriormente excluido de este sistema de integración, en tanto que se refieran a medidas para la aplicación de una acción común o de una posición común, aunque no pueden realizarse en cuestiones con repercusiones militares ni de defensa (27 B TUE). ${ }^{26}$

En su conjunto, el balance de este capítulo parece positivo. Se generaliza la posibilidad de recurrir a este tipo de cooperación en todos los pilares y con un número de países que eventualmente podrá ser de menos de un tercio, y se simplifican las disposiciones. El riesgo de abuso parece equilibrado por la posibilidad que tendrá cualquier Estado singular para pedir la elevación al Consejo Europeo, órgano que reúne la voluntad política suprema en el orden intergubernamental y de la Unión Europea al mismo tiempo.

\section{Cuestiones institucionales}

En el Tratado de la Unión Europea modificado en Amsterdam, las instituciones se contemplaban en la quinta parte del TCE. Las cuestiones que motivaron esencialmente la convocatoria de la CIG, por considerarse que su solución es imprescindible para permitir la ampliación, son fundamentalmente las que aluden al capítulo I (instituciones), secciones primera a tercera (Parlamento Europeo, consejo y comisión). Son las que han sido objeto de mayor debate mediático sobre el número de votos 
asignados en el consejo, la cuantificación de los europarlamentarios correspondientes a cada Estado o de los comisarios en el seno de la Comisión Europea. No estamos ante un mero "baile de números" sino ante el ajuste de la influencia relativa de los Estados ante la perspectiva de una unión ampliada, de equilibrar las diferencias demográficas y el poder de decisión y de garantizar la existencia de un ejecutivo eficiente, al tiempo que de preparar a la Unión Europea para una ampliación sin precedentes. Los objetivos que se pretendían con esta reforma institucional eran ambiciosos. Los resultados son, brevemente, los siguientes:

\section{A. El consejo}

El consejo está contemplado en el derecho comunitario primario, fundamentalmente en los artículos 202 a 210 del TCE, de los cuales el 207 y 210 se modifican en Niza. Le afectan directamente además el Protocolo sobre la Ampliación, artículo 3o. y las declaraciones 20 y 21.

Es la institución cuya modificación preocupaba más a los Estados miembros. En el texto principal del tratado tan sólo aparece alguna reforma de los artículos dedicados al consejo, como la del artículo 207, 2 in fine que consiste en introducir la mayoría cualificada para el nombramiento del secretario general y del secretario general adjunto, el 210 (mayoría cualificada en la fijación de remuneraciones de ciertos altos cargos institucionales comunitarios), o el 214.2 (sobre la designación del presidente y miembros de la comisión, también por mayoría cualificada).

Los cambios principales se contienen en un protocolo anexo sobre la ampliación de la Unión Europea, que en su artículo 1o. deroga el protocolo sobre las Instituciones en la perspectiva de la ampliación de la Unión Europea de Amsterdam. Las declaraciones 20 y 21 adoptadas por la conferencia inciden también en el futuro funcionamiento de esta institución.

La reponderación de votos en el seno del consejo responde a una cuestión histórica que se venía agravando. La composición de Europa desde sus orígenes, compuesta por "Estados grandes y Estados pequeños" exigía priorizar territorio o población con fines de voto. Ante la disyuntiva, en 1951 y 1957 se optó por sobredimensionar el factor de población en los países menos poblados. En las sucesivas ampliaciones se mantuvo el mismo criterio, a pesar de que se rompió la paridad inicial entre Estados grandes y pequeños. De los primeros tan sólo se adhirió uno más: el Reino Unido. De los segundos se ha pasado de tres a diez. Al 
margen queda España, intermedio para estos fines. En consecuencia, la aparente razón demográfica que sustentaba el modo de ponderación ha ido perdiendo razón de ser. De mantenerse este sistema, las futuras ampliaciones acentuarán el desequilibrio, ya que la enorme mayoría de los países candidatos responden a la clasificación en el grupo de "países pequeños", con la excepción de Polonia que es un "intermedio". Si se mantuviera el sistema de ponderación vigente hasta el Tratado de Amsterdam, se abriría la vía para que las decisiones del consejo estuviesen respaldadas por un porcentaje reducido de la población europea en torno a un $50 \% .{ }^{27}$

El Tratado de Niza introduce modificaciones en el sistema de ponderación. Los Estados pequeños ven su peso específico modificado por $2.6 \%$ y los grandes por $2.9 \%$. España, acogida a una situación singular, ve su peso ampliado por $3.37 \%$, otorgando vigencia a la Declaración del Acta final del Tratado de Amsterdam sobre el Protocolo sobre las Instituciones en la perspectiva de la Ampliación de la Unión Europea. ${ }^{28}$

Las modificaciones, que afectan al sistema de ponderación de votos y al modus operandi de la mayoría cualificada, no entran en vigor hasta el 1o. de enero de 2005 (artículo 3.1 del protocolo sobre la ampliación de la Unión Europea y declaración 21). Frente al sistema de dobles mayorías que era el preferido generalmente por los países pequeños, el Tratado de Niza introduce una nueva reponderación y una triple mayoría:

- A partir de 1 de enero de 2005, el numero de votos oscilará entre los 29 de cada uno de los Estados más poblados a los cuatro votos ponderados asignados a Luxemburgo. La fijación definitiva de porcentajes y umbrales de mayoría dependerá del ritmo de la ampliaciones, por lo tanto se tendrán que ir adaptando proporcionalmente a medida que entren nuevos socios. En un principio, la nueva mayoría cualificada quedará fijada en 169 votos de los 237, frente al sistema actual de 62 sobre 87. ${ }^{29}$ En una Europa de 27 miembros, la mayoría

27 Estas cuestiones son ampliamente tratadas por la doctrina comunitaria, véase entre otros a Best, Edward, “The Treaty...”, cit., nota 2, pp. 2-9, y en particular p. 3.

28 La situación reconocida a España supone el recordatorio que de nuestro país asumió una situación particular en el momento de su adhesión a las comunidades europeas al renunciar a mayor peso en el consejo a cambio de contar, como hacían los grandes, con dos comisarios en la comisión, posibilidad que desaparece como consecuencia de las futuras ampliaciones.

29 En la cumbre de Niza se había fijado en 170 lo que suponía un porcentaje del 71,7\% de los votos frente al actual $71,3 \%$. Un texto provisional revisado de fecha 22 de diciembre redujo la mayoría desde 170 a 169. 
cualificada será de 258 votos sobre un total de 345 (declaración 20, apartado 2, de "ponderación de votos en el consejo). La minoría de bloqueo pasa del actual 88 a 91 votos (máximo porcentaje de 73,4\% de los votos) cuando se hayan incorporado todos los candidatos que ya han iniciado las negociaciones. La mayoría de bloqueo, una vez efectuadas todas las proyectadas, será de 91 votos, lo que imposibilita la unión de tres "grandes" que por sí solos puedan bloquear la adopción de decisiones. En una unión de 27 miembros, cada uno de los países "grandes", como son Alemania, Francia, Reino Unido e Italia podrán aliarse con otros dos de entre ellos, más uno de los pequeños para conseguir una minoría de bloqueo.

- Además, cualquier Estado puede solicitar a través de su representante en el consejo, que se compruebe que la mayoría cualificada representa al menos un $62 \%$ del total de la población, según el nuevo artículo 205 TCE y la declaración número 20. Esta mayoría de población deriva de la demanda de Alemania que pretendía lograr más votos en el consejo que Francia, para reflejar una diferencia de población de unos 22 millones. Esta pretensión no fue aceptada, pero en cambio se introdujo la posibilidad de control de población que favorece al Estado germano.

Por lo que respecta a España, intentó hacer valer su posición especial de Estado mediano, y el hecho es que en el momento de su adhesión aceptó tener sólo ocho votos en el consejo, frente a los 10 de los Estados grandes, a cambio de conseguir dos comisarios. A lo largo de las negociaciones previas al tratado, la posición española fue la de aceptar menos votos que Francia, Alemania, Reino Unido, e Italia únicamente, si los alemanes veían recompensada su mayor población con más votos. Finalmente no fue posible mantener el mismo poder de bloqueo que los grandes, pero sí consiguió mayor incremento proporcional de votos. Todo esto contribuyó a aumentar las sensibilidades de Portugal que, teniendo ya sólo cinco votos comparados con los ocho de España, pasaba a 11 frente a 28. Finalmente se concedieron 12 a Portugal y 27 a nuestro país, que podrá bloquear decisiones si al menos consigue el apoyo de dos países grandes y uno mediano. ${ }^{30}$

30 Estas cuestiones pueden consultarse en los archivos del la CIG'2000 en europa.eu.int, y en Best, Edward, “The Treaty...”, cit., nota 2, p. 4. 


\section{B. La comisión}

En el derecho primario comunitario se encuentra regulada fundamentalmente en los artículos 211 a 219 TCE, de los cuales quedan modificados el 214, 215 y 217 del TCE en su versión de Niza. Además, por lo que respecta al nombramiento de sus miembros le afecta la modificación del artículo 210 sobre el consejo. Resulta también afectada por el Protocolo sobre la ampliación de la Unión Europea, en su artículo 4o., y por las declaraciones 20 y 21 .

En el Tratado de Amsterdam se preveía que las modificaciones en su seno quedarían vinculadas a las modificaciones que se produjesen en el consejo. ${ }^{31}$ Se partía de la problemática derivada de las futuras ampliaciones y en particular de la necesidad de poner un límite en el número de comisarios para evitar que esta institución se convierta en una "asamblea de delegados nacionales". En Niza, los quince han decidido que a partir del 1o. de enero de 2005, los Estados que en la actualidad tienen dos comisarios (Alemania, Francia, Reino Unido, Italia y España) perderán uno de ellos. Cada nuevo país miembro contará con un comisario. Cuando el colegio alcance los 27 miembros, los Estados tendrán que decidir por unanimidad cuál deba ser el número (siempre inferior a 27) en que quedará fijado el número de miembros de la comisión, según el protocolo, sobre la ampliación de la Unión Europea. Cuando se produzca dicha decisión unánime, el número de miembros de la comisión será inferior al número de Estados. Estos comisarios serán elegidos con arreglo a "una rotación igualitaria" basada en la estricta equiparación entre los Estados, tanto por lo que respecta a los turnos, como por la permanencia en el cargo, y reflejando la diversidad demográfica y geográfica de la Unión Europea. Esta composición refleja el deseo de los países miembros más pequeños que prefieren una comisión fuerte e independiente como garantía frente al poder de los grandes.

31 Protocolo sobre las Instituciones en la perspectiva de la Ampliación de la Unión Europea, derogado en Niza por el Protocolo sobre la Ampliación Europea. En Amsterdam se acordó que llegado el momento de la primera ampliación después del Tratado de Amsterdam, la comisión comprenderá un nacional de cada uno de los Estados miembros, siempre que para esa fecha se haya modificado la ponderación de votos en el consejo de forma aceptable para todos los Estados miembros mediante una nueva ponderación de votos o bien mediante una doble mayoría, teniendo en cuenta todos los aspectos pertinentes, en especial la compensación a aquellos Estados miembros que renuncien a la posibilidad de designar un segundo miembro de la comisión. 
La presencia de un comisario por Estado se entiende como un modo de que todos los intereses se tengan en cuenta, ya que la opción de reducir la comisión se encontró con la oposición, manifestada en la cumbre informal de Biarritz, ${ }^{32}$ de los que temían que la reducción en tamaño significase su vaciado de Poder Ejecutivo para convertirse en un mero órgano administrativo.

El Tratado de Niza refuerza, en la línea iniciada en Amsterdam, los poderes del presidente de la comisión. El nombramiento pasa, de ser responsabilidad de los Estados miembros, a corresponder - por mayoría cualificada - al máximo órgano jerárquico de la Unión Europea, es decir, al Consejo Europeo. Se adopta así un sistema de elección, al menos nominalmente institucional, y no estrictamente intergubernamental. El nombramiento del resto de los miembros corresponderá al Consejo de Ministros de común acuerdo con el presidente. El Parlamento aprobará colegiadamente las propuestas, y finalmente tanto el presidente como el resto de los miembros son nombrados por mayoría cualificada por el consejo. Este es un ámbito en el que se ha avanzado, al abandonarse la regla de la unanimidad.

El presidente queda reforzado también, porque no sólo puede fijar la orientación política de la comisión, sino que además le corresponde decidir su organización interna. Es competencia suya estructurar y repartir las responsabilidades entre los miembros de la comisión que ejercen su cargo bajo su autoridad. Sus poderes políticos se refuerzan porque puede forzar, previa aprobación del colegio, la dimisión de un comisario.

\section{El Parlamento Europeo}

El Parlamento Europeo es la institución que menos cambios estructurales ha sufrido. En el derecho primario comunitario se encuentra regulado en el cuerpo principal del Tratado CE, fundamentalmente en los artículos 189 a 201, además del Protocolo sobre la ampliación y las declaraciones 20 y 21 del Tratado de Niza.

En Amsterdam se había acordado un número máximo de europarlamentarios (700), que es ahora superado para pasar a 732 en el Tratado de

32 Las conclusiones de la cumbre de Biarriz están disponibles en la sección dedicada al Consejo Europeo, dentro del servidor europa.eu.int. Asimismo están directamente enlazados en muchas páginas $W e b$ de los Centros de Documentación Europea de la Unión, entre otros en http://www.usc.es/cde, véase documentos de interés. 
Niza. ${ }^{33}$ Puede afirmarse, en términos generales, que el Parlamento ha visto reafirmado su papel institucional. La superación del número de miembros del Parlamento se debe en gran medida a las negociaciones que tuvieron lugar respecto del equilibrio franco-alemán. La diferenciación entre estos dos países respecto de su representación parlamentaria ya se inició en 1992 con el Tratado de Maastricht. En Niza, Alemania mantiene sus 99 representantes, como compensación por la paridad de votos en el consejo con Francia. El resto, los "grandes" pasan de tener 81 parlamentarios a 72. Bélgica, Portugal y Grecia reciben mayor representación a cambio de concesiones en el consejo, frente a la República Checa y Hungría, que con población semejante obtienen derecho a menos europarlamentarios.

Otras reformas introducidas por Niza refuerzan el papel institucional del Parlamento. Entre ellas está la consolidación de los partidos políticos europeos en el artículo 191, TCE, y la declaración correspondiente al mismo. El estatuto de los partidos políticos europeos será establecido por el procedimiento de codecisión, al igual que las normas relativas a su financiación a cargo de la comunidad, y totalmente independiente de la de los partidos políticos en el nivel nacional.

Niza refuerza el papel institucional del Parlamento a través de la extensión de la adopción de decisiones por el procedimiento de codecisión, aunque no se impone de forma universal.

Tal vez la reforma principal sea la legitimación activa para recurrir en anulación (artículo 230, TCE) ante el Tribunal de Justicia de Luxemburgo en las mismas condiciones que el Parlamento, el consejo, y los Estados miembros, y la legitimación activa para solicitar al Tribunal de Justicia que se defina sobre la compatibilidad con el tratado de los acuerdos internacionales que se vayan a celebrar con terceros Estados u organizaciones.

\section{Tribunal de Justicia}

El nivel del derecho comunitario primario se encuentra contemplado fundamentalmente en el cuerpo principal del tratado, en la quinta parte, título primero, capítulo primero, sección cuarta, artículos 220 a 245; ade-

33 La ampliación del número máximo de eurodiputados se debe a la presión ejercida por Alemania para hacer valer su mayor peso demográfico, manteniendo sin variar su actual número de 99 eurodiputados. Esto se utilizó como moneda de cambio a la ponderación de votos en el seno del consejo y para mantener la paridad entre Francia (con menor peso específico) y Alemania. 
más en el protocolo sobre su Estatuto. En Niza quedan alterados los artículos 220, 221, 222, 223, 224, 225 y 245, así como el Protocolo sobre su Estatuto. Se insertan dos nuevos artículos: el $225 \mathrm{~A}$ y el 229 A.

Pese a que su modificación no fue objeto de tanta atención de los medios de comunicación como la de las instituciones anteriormente citadas, es la que mayores y más profundas transformaciones ha sufrido con el Tratado de Niza, al menos en potencia, ya que el tratado, citando las posibles modificaciones de funcionamiento, remite la implantación definitiva de la mayoría de ellas a una decisión unánime del consejo, que modificando el Estatuto determinará su alcance y contenido concreto.

Las reformas parten del sistema empleado para la regulación del funcionamiento del tribunal:

- Se modifica el Protocolo sobre su Estatuto y muchas de las cuestiones que antes se regulaban en el propio tratado ahora pasan a formar parte del Estatuto, cuya reforma (excepto por lo que se refiere al primer título del Estatuto) no requiere de una nueva Conferencia Intergubernamental y posterior ratificación por los Estados, ${ }^{34}$ sino que podrá tener lugar mediante una votación unánime del consejo.

- Se introduce mayor flexibilidad en la modificación o reforma de los Reglamentos de Procedimiento del Tribunal de Justicia y del Tribunal de Primera Instancia, siendo sustituidas la unanimidad por la mayoría cualificada en el seno del consejo. Esta flexibilización toma en cuenta importantes estudios que se habían presentado con anterioridad a las negociaciones para la reforma. ${ }^{35}$

El Tratado de Niza introduce una nueva arquitectura judicial europea. Convierte al Tribunal de Primera Instancia en un "juez común" comunitario, permite crear salas jurisdiccionales, y establece que el TJCE pase

34 Este procedimiento utilizado hasta la entrada en vigor del Tratado de Niza, no afecta sin embargo al título III del Estatuto dedicado al procedimiento.

35 Entre estos estudios destacan algunos como el Documento de Reflexión sobre el Futuro del Sistema Jurisdiccional de la Unión Europea presentado por el presidente del Tribunal de Justicia ante los ministros de Justicia reunidos en Consejo de Ministerios en Bruselas el 27 y 28 de mayo de 1999; el Informe del Grupo de Reflexión sobre el Futuro del Sistema Judicial de las Comunidades Europeas de enero de 2000, elaborado bajo el antiguo presidente del tribunal señor Due para la comisión, y que a su vez a ésta le sirvió para elaborar su Contribución Complementaria a la Conferencia Intergubernamental sobre las Reformas Institucionales-Reforma del Sistema Jurisdiccional Comunitario (1o. de marzo de 2000) o la Contribución del Tribunal de Justicia y del Tribunal de Primera Instancia a la Conferencia Intergubernamental de 25 de febrero de 2000. 
como órgano supremo, distinto del TPI. Según la redacción del artículo 220 del TCE: "El Tribunal de Justicia y el Tribunal de Primera Instancia garantizarán, con arreglo a sus respectivas competencias, el respeto del derecho en la interpretación y en la aplicación del tratado pudiendo agregarse salas jurisdiccionales que ejerzan competencias jurisdiccionales previstas en el tratado".

La composición del Tribunal de Justicia queda fijada, de acuerdo con el nuevo artículo 221 de "un juez por cada Estado miembro". Sigue sin exigirse la nacionalidad, aunque probablemente se mantendrá el pacto tácito conforme al cual habrá un juez nacional de cada país miembro de la Unión Europea. De esta manera se evita la necesidad de reformas numéricas que se originan en la Unión Europea, cada vez que se adhiere un nuevo Estado. ${ }^{36}$ En cuanto al Tribunal de Primera Instancia, deberá contar con al menos un juez cada Estado miembro, remitiendo a la concreta fijación en el Estatuto.

La figura del abogado general sufre ciertas modificaciones. Frente a la situación actual en la que su intervención es preceptiva, el Tratado de Niza remite al Estatuto la determinación de aquellos asuntos en los que sea necesaria la participación de estos juristas. En general intervendrá excepto si el asunto no plantea ninguna cuestión nueva de derecho, en cuyo caso, el TJCE podrá decidir, una vez escuchado el abogado general que el asunto sea juzgado sin las conclusiones de este. Además remite a la modificación del Estatuto la posibilidad de que el TPI sea asistido por Abogados generales. El Estatuto aprobado en Niza se limita a mantener la situación existente, que consiste en que los propios jueces puedan desempeñar la función de abogado general, pero la citada vía introducida para la modificación del Estatuto permite cambios posteriores si se produce una votación unánime del consejo.

Otras modificaciones se refieren a la organización del tribunal. Con el Tratado de Niza la regla general pasa a ser el funcionamiento en salas frente al sistema anterior en que la regla general era el pleno. No obstante, si un Estado miembro o una institución que sean parte en el proceso lo solicitan, el tribunal decidirá en pleno. De acuerdo con el nuevo Estatuto el modo ordinario será el funcionamiento en salas de tres o cinco jueces, en "gran sala" de 11 jueces o en pleno. En cuanto al recurso al pleno,

36 Esta reforma es fruto de la recepción de lo señalado por el Tribunal de Justicia en su Informe del Tribunal de Justicia sobre Determinados Aspectos de la Aplicación del Tratado de la Unión Europea (1995). 
además de la solicitud de las partes a los que hemos referido, puede utilizarse en supuestos señalados en el Tratado CE como los artículos 195.2, 213, 216 y 247.7; o cuando (según dispone el artículo 16 del Estatuto) "considere que un asunto del que conozca reviste una importancia excepcional... una vez oído el Abogado General....".

Las salas jurisdiccionales constituyen la mayor novedad en la reforma institucional del sistema jurisdiccional. Podrán crearse a través de decisiones del consejo a propuesta de la comisión y previa consulta de Tribunal de Justicia y del Parlamento Europeo. También pueden crearse por petición del tribunal y previa consulta a la comisión y al Parlamento Europeo. Sus miembros serán designados por el consejo por unanimidad entre "personas que ofrezcan absolutas garantías de independencia y que posean la capacidad necesaria para el ejercicio de funciones jurisdiccionales". Una declaración de la conferencia insta a la comisión a preparar una decisión para la creación de una sala jurisdiccional competente para resolver en primera instancia litigios entre la comunidad y sus agentes.

Se abre la posibilidad de alterar las competencias del tribunal a través de futuras modificaciones del Estatuto. Según el nuevo artículo 225.1 El Tribunal de Primera Instancia será competente para conocer en primera instancia de los recursos contemplados en los artículos 230 (anulación), 232 (omisión), 235 (indemnización), 236 (función pública) y 238 (cláusula contractual compromisoria), y cualquiera otro que pueda señalar el Estatuto excepto los que se reserven a una sala jurisdiccional y los que el Estatuto, reserve al Tribunal de Justicia. Esto significa que el TPI pasa a tener una competencia general como juez común en un sistema jurisdiccional en el que la doble instancia pasa a ser la regla general, como garantía hacia los litigantes, incluso cuando éstos sean Estados o instituciones comunitarias. ${ }^{37}$ Algunos recursos se mantienen como competencia exclusiva del TJCE como el recurso por incumplimiento sobre la compatibilidad de la actividad nacional con la comunitaria.

Las decisiones de las eventuales salas jurisdiccionales podrán ser impugnadas en casación (por motivos de derecho) o mediante un recurso de apelación (por motivos de hecho) ante el TPI. Las decisiones de este último tribunal, resolviendo recursos frente a decisiones de las salas podrán

37 Sobre estas cuestiones, es de gran interés Alonso García, R., "Principios e institucionais e políticos da Comunidade: o Tratado de Niza", Xçornada sobre o dereito administrativo comunitario, Santiago de Compostela, Escola Galega de Adminitración Pública, 22 de febrero de 2001. 
ser objeto de ulterior recurso ante el TJ por motivos excepcionales, y para garantizar la unidad y la coherencia del derecho comunitario.

Otra modificación potencial introducida en Niza se refiere a las cuestiones prejudiciales del artículo 234, que podrán dejar de ser de competencia exclusiva del TJ, para poder ser conocidas por el TPI, en virtud del artículo 225.3, que remite una vez más a la futura reforma del Estatuto, y siempre que el propio TPI no decida remitir la cuestión directamente al $\mathrm{TJ}$, es decir, sin entrar a conocerla, en aras de una mayor coherencia y unidad del sistema comunitario.

La declaración sobre el artículo 225 recoge la invitación de la conferencia a que tanto el tribunal como la comisión efectúen un estudio global del reparto de competencias y a que presenten propuestas para examinarlas cuando entre en vigor el Tratado de Niza.

Otras instituciones y órganos han sido objeto de alguna reforma de menor calado. Así por ejemplo se fija en 350 el número máximo de miembros del Comité Económico y Social y del Comité de las Regiones; se introduce la posibilidad de modificación de ciertas disposiciones de los Estatutos del Banco Europeo de Inversiones a través de la decisión unánime del consejo, se establece la creación de un Comité de Protección Social de carácter consultivo para fomentar la protección social, y se fija Bruselas como la futura sede de reuniones del Consejo Europeo, como ya indicábamos.

\section{Sistemas de adopción de decisiones}

\section{A. Ampliación el procedimiento de codecisión}

El Tratado de Niza amplía la utilización del procedimiento de codecisión en la adopción de decisiones, aunque sin llegar a imponer esta vía como la general en el procedimiento legislativo. La codecisión es un procedimiento introducido en el Tratado de Maastricht y simplificado en el Tratado de Amsterdam que constituye la vía que garantiza mayor capacidad de influencia sobre la legislación comunitaria al Parlamento Europeo en la adopción de decisiones. Se regula en el artículo 251 del TCE y constituye una profundización en el procedimiento de cooperación. El consejo no puede imponer su decisión, en caso de desacuerdo con el Parlamento en tercera lectura respecto del texto redactado por el Comité de Conciliación. 
Niza extiende la decisión por este sistema. En el artículo 13 TUE (prohibición de toda discriminación), sometido en Amsterdam a unanimidad y consulta al PE para la adopción de decisiones de lucha contra la discriminación, se añade un párrafo segundo en virtud del que, cuando se trate de medidas de apoyo de medidas nacionales (excluida la armonización) se decidirá por codecisión.

Se introduce también la codecisión en virtud de un nuevo apartado que se añade con el número de 5 al artículo 67 , a las medidas previstas en el artículo 63, 1 (asilo, refugiados y desplazados con arreglo a las correspondientes convenciones y acuerdos internacionales) y 63,2 a) sobre normas mínimas para conceder protección temporal a las personas desplazadas procedentes de terceros países que no puedan volver a su país de origen y para personas que por otro motivo precisen de protección internacional. Igualmente pasar a decidirse por el método de codecisión las medidas del artículo 65, TCE, que se adopten de conformidad con el 67, TCE, a excepción de las relativas al derecho de familia, es decir las medidas sobre cooperación judicial en materia civil con repercusión transfronteriza, necesarias para el correcto funcionamiento del mercado interior (notificación y traslado de documentos judiciales y extrajudiciales, cooperación en la obtención de pruebas, etcétera.)

\section{B. Reducción del ámbito de la unanimidad}

Los resultados en este ámbito parecen positivos en tanto que se amplía el numero de cuestiones que se decidirán por mayoría, si bien es cierto que la agenda que había propuesto la comisión había fijado ciertos sectores que consideraba prioritarios para la extensión de la mayoría y que sin embargo no han sido afectados en ese sentido. En concreto han quedado excluidos la fiscalidad (artículos 93, 94 y 175 del TCE), la política social (42 y 137, TCE), y la seguridad social. El Reino Unido fue uno de los principales detractores que evitó abandonar la regla de la unanimidad.

El asilo y la inmigración podrán decidirse por mayoría, pero sólo después del año 2004 (en virtud del Protocolo sobre el artículo 67), así como los asuntos relativos a la cohesión que han quedado aplazados hasta el año 2007 (con dos declaraciones contradictorias de las que tomó nota la conferencia, según hemos visto).

La ampliación en Niza de los ámbitos en los que las decisiones podrán ser mayoría es evidente, sin perjuicio de que ha sido calificada por 
algunos de insuficiente, ya que a medida que se vayan produciendo nuevas ampliaciones, se incrementará la dificultad de abandonar tal unanimidad. ${ }^{38}$ Destacamos algunos de los ámbitos (en total son unos 50) en los que se pasa a adoptar la mayoría cualificada como consecuencia del Tratado de Niza:

- El artículo 18.2 TCE en su redacción de Amsterdam exigía la unanimidad (en un procedimiento de codecisión) para la adopción de decisiones sobre la ciudadanía. El nuevo párrafo 2 del artículo 18, TCE, excluye la necesidad de la unanimidad, manteniendo el sistema de adopción de decisiones por el método de codecisión, excepto (nuevo párrafo 3) por lo que respecta a pasaportes, documentos de identidad, permisos de residencia o documentos asimilados, disposiciones referentes a la seguridad social o a la protección social.

- Otro ámbito en el que se introduce la regla de la mayoría cualificada frente al de la unanimidad es de la adopción de medidas adecuadas a la situación económica para paliar la aparición de dificultades graves en el suministro de productos, así como las medidas que puede adoptar el consejo para hacer frente a las dificultades graves actuales o potenciales en un Estado miembro ocasionadas por acontecimientos excepcionales. Pueden consistir en medidas adecuadas a la situación económica o incluso una ayuda financiera al Estado en cuestión, de acuerdo con el artículo 100 del TCE.

- La representatividad de la comunidad en el ámbito internacional en relación con sus competencias sobre la coordinación de las políticas económicas la política monetaria (artículo 111), pasa también a decidirse por mayoría. La adopción de medidas tendentes a la rápida introducción de la moneda única, al margen de la fijación de paridades (artículo 123.4).

- Las decisiones en materia de política comercial del artículo 133 por lo que se refiere, en particular, a los acuerdos internacionales en el ámbito del comercio de servicios y de los aspectos comerciales de la propiedad intelectual, según el artículo 133 (siempre que tales acuerdos no se refieran asuntos en los que la regla general de adopción de decisiones comunitarias sea la unanimidad, o siempre que

38 Fonseca Morillo, F. J., "Las transformaciones institucionales: el consejo y la comisión”, en Faramiñán Gilabert, J. M. (coord.), Reflexiones en torno al Tratado de Amsterdam y el futuro de la Unión Europea, Granada, 2000. 
no se trate de materias en las que la comunidad aún no ha ejercicio sus competencias, cuando se refiera a disposiciones que excedan las competencias comunitarias, y las normas sobre los tratados en materia de transportes).

Como se ve, se han producido algunos avances, que no han logrado erradicar el recurso a la unanimidad, pero que sí constituyen una progresión en la extensión de la regla de la adopción de decisiones por mayoría.

\section{PERSPECTIVAS}

El tratado aprobado en Niza ha sido criticado por muchos, y rechazado por 76.014 irlandeses, quienes han dicho "no" al proceso de ratificación. No obstante, parece ser el único texto capaz de obtener un acuerdo por parte de los quince en la Conferencia Intergubernamental de 2000. ¿Hubieran debido llegar más lejos?, ¿debería haberse abandonado definitivamente la unanimidad?, ¿cuál será la solución a los problemas que se puedan plantear con la ratificación?

Estas y otras cuestiones que han suscitado las principales críticas, consisten en la expresión de deseos casi siempre fundados en meras teorías, que auguran un porvenir más próspero si se acelera el proceso de ampliación.

El resultado de Niza es, al final de las cuentas, un añadido más al proceso de creación de una Unión Europea "sui generis" iniciado en 1992 en Maastricht. Y de otro iniciado en Luxemburgo en 1993 y reforzado en Helsinki en 1999: es decir, la ampliación. Las reticencias encontradas a la hora de ratificar aquel tratado que eventualmente entró en vigor en 1993 (negativa de Dinamarca a una ratificación en un primer referéndum, resoluciones contrarias de algunos Tribunales Constitucionales, particularmente alemán, etcétera) son perfectamente conocidas por todos. Los avatares de las negociaciones de la ampliación parecen ocupar menos la atención general (que no la de los expertos), sin que por ello sean menos complejas. Algunos aspectos, como la incidencia en el reparto de fondos estructurales y de cohesión, los periodos transitorios para la libre circulación de ciudadanos del este, o para la adquisición de inmuebles por parte de los actuales miembros en los países candidatos, sí que han alcanzado la opinión pública y han orientado los discursos de los principales dirigentes europeos que se han manifestado respecto del futuro de Europa. 
El de Niza es un tratado que, en cuanto que es un texto técnicamente ideado para formular una reforma que permita la ampliación, logra formalmente tal objetivo. Ha consolidado además la estructura en pilares de una unión, y abre en su Declaración 23 un debate generalizado sobre el futuro de Europa, cuyo lanzamiento corresponde a la Presidencia sueca y a la belga asistidas por el Parlamento Europeo y por la comisión. Este debate ya se ha iniciado y abarca muchas y muy importantes cuestiones, más allá de las reformas institucionales y la jerarquía normativa.

Parece que una vez más no estamos ante un texto con vocación de permanencia sin modificaciones. Es posible que, al igual que ocurriera con el límite de 700 europarlamentarios fijados por el Tratado de Amsterdam, la conferencia de 2004 y los tratados de adhesión modifiquen algunos de los acuerdos de Niza, muchos de los cuales, como la reponderación de votos, no entrarán en vigor, en ningún caso, antes de 2005.

Las soluciones que parecen previsibles a la situación irlandesa (una renegociación general, un nuevo referéndum después de establecer cláusulas especiales para Irlanda, que como país neutral puede tener reticencias para aceptar todas las disposiciones relativas a las PESC) se unen al resto de las que se están debatiendo estos días en el marco del debate abierto de conformidad con la citada Declaración 23: la conveniencia de una Constitución europea, que no es apoyada por los británicos pero en cambio sí es favorecida con diversos matices por Francia y por el ministro alemán de Asuntos Exteriores o por Portugal; la profundización en las cooperaciones reforzadas, apoyada por Chirac; las diversas posibilidades de establecer una segunda cámara legislativa en el marco de la Unión Europea que, según Schoröder, debería ser el propio consejo; o estar constituido por los parlamentos nacionales, según Jospín; o la necesidad de un gobierno económico, como la apuntada por Romano Prodi, entre otras.

Estamos, o seguimos estando ante la disyuntiva de ampliación o profundización que ya se preveía en 1992. En realidad, una ampliación que no vaya acompañada de mayor profundización en la integración resultará poco efectiva y no posibilitará grandes avances en la construcción europea. Al mismo tiempo, las cuestiones abiertas tienen tal trascendencia que merecen la dedicación de tiempo y debate. Resulta interesante, y comprometido para España, que muchas de estas negociaciones se desarrollarán durante el primer semestre de 2002, que corresponde a la Presidencia española, que deberá liderar el debate y alentar el hallazgo de soluciones, reto cuya resolución corresponde a todos los europeos. 